\title{
Fracture mechanics determine the lengths of interface ruptures that mediate frictional motion
}

\author{
Elsa Bayart, Ilya Svetlizky and Jay Fineberg*
}

The transition from static to sliding friction is mediated by rapid interfacial ruptures $^{1-5}$ propagating through the solid contacts forming a frictional interface ${ }^{6}$. While propagating, these ruptures correspond to true shear cracks ${ }^{7}$. Frictional sliding is initiated only when a rupture traverses the entire interface'; however, arrested ruptures can occur at applied shears far below the transition to frictional motion ${ }^{8-17}$. Here we show, by measuring the real contact area and strain fields near rough frictional interfaces, that fracture mechanics quantitatively describe rupture arrest and therefore determine the onset of overall frictional sliding. Our measurements reveal both the local dissipation and the global elastic energy released by the rupture. The balance of these quantities entirely determines rupture lengths, whether finite or system-wide. These results confirm a fracture-mechanics-based paradigm ${ }^{7,15,18}$ for describing frictional motion and shed light on the selection ${ }^{18-21}$ of an earthquake's magnitude.

A frictional interface is formed by the interlocked solid asperities of rough surfaces in contact, whose area is much smaller than the nominal one ${ }^{6}$. Failure of the asperities via rupture fronts is the fundamental mechanism responsible for the transition from static to sliding frictional motion ${ }^{1,4}$. Ruptures can propagate well before the onset of global sliding, and then arrest before spanning the entire interface. Rupture arrest can, for example, result from inhomogeneous stress distributions along the interface ${ }^{8-10}$. As no overall motion of the contacting bodies is induced by such events, they are often called precursors to sliding motion. Arrested events are analogous to earthquakes, which are dynamic ruptures of finite extent within pre-existing natural faults; the boundary between contacting tectonic plates ${ }^{22,23}$. Predicting the length of precursory ruptures is, therefore, closely related to the question of what determines the size of an earthquake ${ }^{18-21}$

Since their initial discovery ${ }^{8}$, a rich variety of models has been dedicated to the dynamics of precursory ruptures in frictional systems. Aimed at reproducing nucleation and arrest, these include minimalistic one-dimensional (1D) models ${ }^{9}$, discrete contacts descriptions ${ }^{11,13,16}$, rate-and-state friction laws ${ }^{17}$ and fracture mechanics ${ }^{12}$. These models are able to reproduce the existence of arrested ruptures but they provide no explicit predictions of where and how arrest occurs in real systems. Recent theoretical work $^{15}$ explained the available data ${ }^{8}$ by explicitly demonstrating how fracture mechanics can be used to predict rupture arrest. Here we describe new experiments that confirm these theoretical predictions and show that this general framework indeed enables us to understand the selection of rupture length for any system geometries and loading conditions.

Recent experiments have shown that the strain fields driving ruptures along frictional interfaces are described ${ }^{7}$ by the linear elastic fracture mechanics (LEFM; ref. 24). In this context, a precursory event is an arrested $\mathrm{crack}^{12,15,18}$. In fracture mechanics, crack arrest is defined by the Griffith criterion $^{24}$, a crack arrests when the amount of energy flowing to its tip becomes smaller than the fracture energy $\Gamma$, the dissipated energy per unit crack advance. Following the theoretical approach outlined in earlier studies ${ }^{15,18}$ we will experimentally verify that this criterion is fulfilled by spontaneously arrested frictional ruptures. These results provide new insights into the predictability of both frictional processes and earthquake dynamics.

We examine the frictional sliding of two poly(methylmethacrylate) (PMMA) blocks whose contact interface is flat to within a few $\mu \mathrm{m}$. We define $x, y$ and $z$ as, respectively, the rupture propagation, normal loading and sample thickness directions. We use two different sample geometries (Fig. 1a). The asymmetric geometry is formed by blocks of different thicknesses $(6 \mathrm{~mm}$ top block, $30 \mathrm{~mm}$ bottom block) and dimensions with a $3 \mu \mathrm{m}$ r.m.s. surface roughness along the interface. The symmetric geometry is made of blocks having the same dimensions whose interface is formed by two optically flat surfaces. PMMA has a strainrate-dependent Young's modulus $3<E<5.6 \mathrm{GPa}$ and Poisson ratio $v=1 / 3$. The Rayleigh wave speeds are $c_{\mathrm{R}}=1,237 \pm 10 \mathrm{~m} \mathrm{~s}^{-1}$ (plane stress) and $c_{\mathrm{R}}=1,255 \pm 10 \mathrm{~m} \mathrm{~s}^{-1}$ (plane strain) (Methods). The blocks are pressed together with an externally imposed normal force $F_{\mathrm{N}}$, ranging from 1,500-7,000 N. After applying $F_{\mathrm{N}}$, shear forces $F_{\mathrm{S}}$ are applied either at a single point or more uniformly via a sliding stage (Fig. 1a), giving rise to a variety of inhomogeneous distributions of normal and shear stresses along the interface (see examples in Fig. 1b). Throughout each experiment, continuous parallel measurements of the $2 \mathrm{D}$ strain tensor $\varepsilon_{i j}(t)$ are performed at 15 to 18 locations along and $3.5 \mathrm{~mm}$ above the interface at a rate of $10^{6}$ samples per second (Fig. 1a). From the strain measurements we obtain stresses, while taking into account material viscoelasticity (Methods). At the same time, we measured the real contact area $A(x, t)$ at $1,280 \times 8(x \times y)$ spatial locations along the interface at 580,000 frames per second by an optical method based on total internal reflection ${ }^{1,4,7}$. Under these stress conditions, we observe a succession of arrested ruptures of increasing length (Fig. 1c), well before $F_{\mathrm{S}}$ reaches the threshold for overall stick-slip motion of the blocks (Fig. 1d). The transition to the overall motion of the contacting blocks happens only when these ruptures span the entire interface (event VI).

The variations of the stresses at the tip of each propagating rupture are quantitatively described by the singular fields predicted by LEFM for shear (mode II) cracks $^{7}$ (Fig. 2a). The singular term of the stress field has the form ${ }^{24}$ :

$$
\Delta \sigma_{i j}(r, \theta)=\frac{K_{\mathrm{II}}(v)}{\sqrt{2 \pi r}} \Sigma_{i j}^{\mathrm{II}}(\theta, v)
$$


a

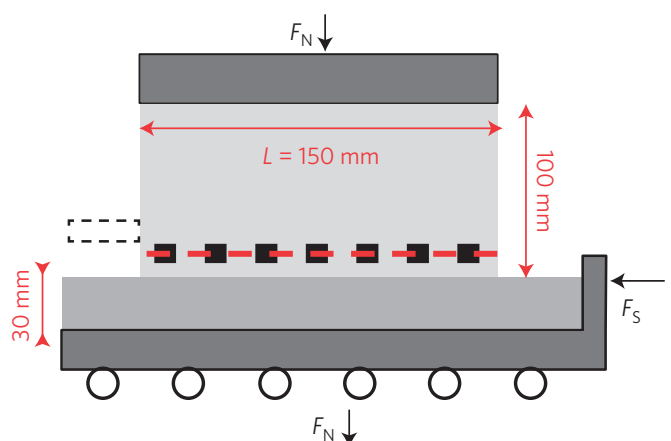

$F_{N} \downarrow$

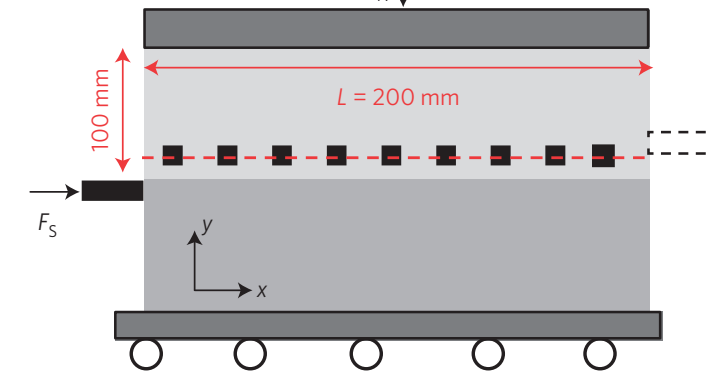

c

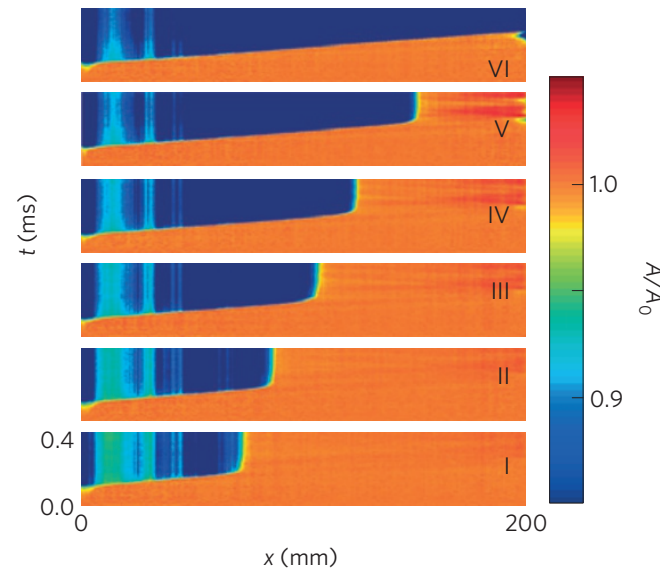

b
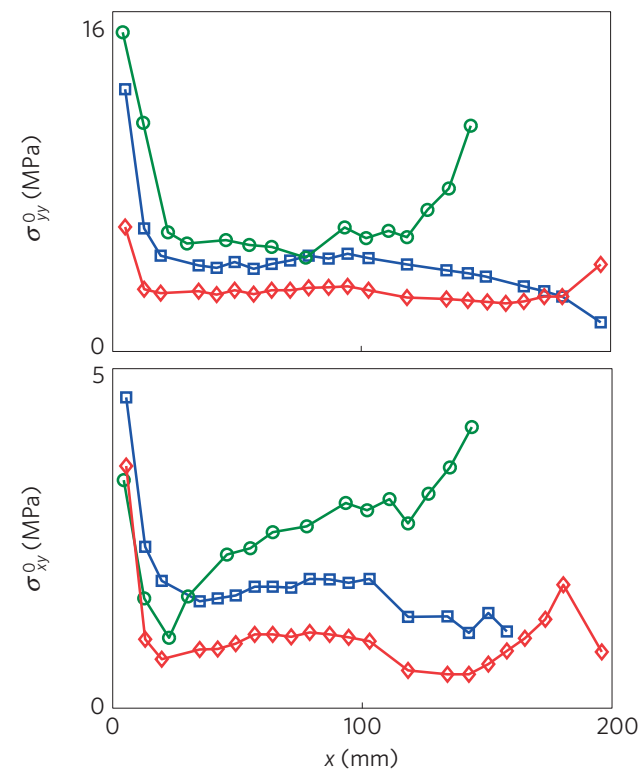

d

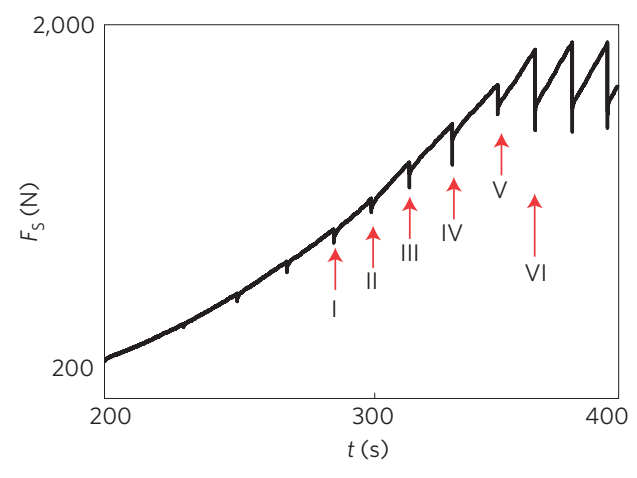

Figure 1 | Experimental set-up and the definition of spontaneously arrested interfacial ruptures. a, Asymmetric (top) and symmetric (bottom) system geometries with respective thicknesses of top and bottom blocks of $6 \mathrm{~mm}$ and $30 \mathrm{~mm}$ (asymmetric) and $6 \mathrm{~mm}$ (symmetric). Shear forces, $F_{S}$, are applied homogeneously through a rigid stage (top) or at a single point on the trailing edge of the block (bottom). An immobile stopper that locally fixes the displacement of the top block was optionally used (dashed pin in figure). An array of between 15 to 18 strain gauge rosettes measures the three components of the 2D-strain tensor $3.5 \mathrm{~mm}$ above the interface (Methods). $\mathbf{b}$, Examples of normal stress, $\sigma_{y y}^{0}$, and shear stress, $\sigma_{x y}^{0}$, distributions along the interface before a sliding (system-wide) event are represented, each corresponding to a different loading configuration: homogeneous loading with a stopper for the asymmetric set-up $\left(F_{N}=4,200 \mathrm{~N}\right.$ ) (green circle), single-point loading both without $\left(F_{\mathrm{N}}=4,500 \mathrm{~N}\right)$ (blue square) and with a stopper $\left(F_{\mathrm{N}}=3,500 \mathrm{~N}\right)$ (red diamond) for the symmetric set-up. c, Spatio-temporal evolution of the contact area $A(x, t)$ for successive events I-VI in a typical experiment (blue curve in $\mathbf{b}$ ). The measurements are normalized by the contact area at the initial time $A_{0}=A(x, 0)$ immediately before each event. Time slices of $0.4 \mathrm{~ms}$ duration are presented for each event. The presented ruptures propagate at velocities $0.80<v<0.95 c_{R}$. $\mathbf{d}$, The increase in applied shear force $F_{\mathrm{S}}$ in time for the experiment described in $\mathbf{c}$. The arrows denote the precursory events I-V together with the system-wide event $\mathrm{VI}$.

in polar coordinates with respect to the crack tip, where $\Sigma_{i j}^{\mathrm{II}}(\theta, v)$ is a known universal angular function and $K_{\mathrm{II}}$ is the stress intensity factor. $\Delta \sigma_{i j}$ expresses the stress changes ${ }^{25}$ between the initially applied and residual stresses along the frictional crack faces. In the framework of LEFM no generality is lost by adding constant values, $\sigma_{x x}^{0}, \sigma_{y y}^{0}$ and $\sigma_{x y}^{\text {res }}$ (Fig. 2a) to equation (1) (Methods). For a known rupture velocity $v$, the amplitude of the singular term - that is, the stress intensity factor $K_{\mathrm{II}}$-is related to the energy release rate $G$, defined as the flux of elastic energy per unit extension of a crack's tip, by:

$$
\mathrm{G}=\frac{\alpha}{E} f_{\mathrm{II}}(v) K_{\mathrm{II}}^{2}(l, v) \underset{v \rightarrow 0}{\longrightarrow} G_{\text {stat }}(l)
$$

where $f_{\text {II }}(v)$ is a universal function, whose value is nearly unity for low rupture velocities $v$. The coefficient is $\alpha=1$ for the plane stress conditions used in the symmetric system, whereas $\alpha=\left(1-v^{2}\right)$ for the plane strain conditions used in the asymmetric system ${ }^{7}$. Following equation (1), fitting the three stress components (see, for example, Fig. 2a) provides a dynamic measurement ${ }^{7}$ of $K_{\mathrm{II}}$.

During rupture propagation, $G=\Gamma$. Using the value of $K_{\mathrm{II}}$ in equation (2), we can therefore calculate the fracture energy $\Gamma$, which has been shown to be roughly independent of velocity ${ }^{7}$. As Fig. 2b shows, we find that $\Gamma$ depends linearly on the mean normal stress, $\left\langle\sigma_{y y}\right\rangle$. If we assume that the real contact area, $A$, entirely determines $\Gamma$, this result is in accordance with both the Bowden and Tabor picture $^{6}$ ( where $A \propto F_{\mathrm{N}}$ ) and the suggestion ${ }^{7}$ that $\Gamma$ effectively measures the number of contacts that have to be broken in order for a crack to propagate.

Can a frictional rupture arrest be described by the fracturemechanics-based criterion for crack arrest? If so, according to 
a

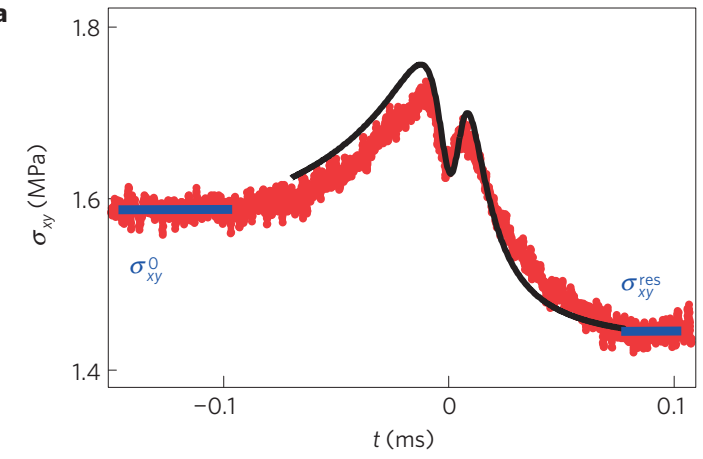

C

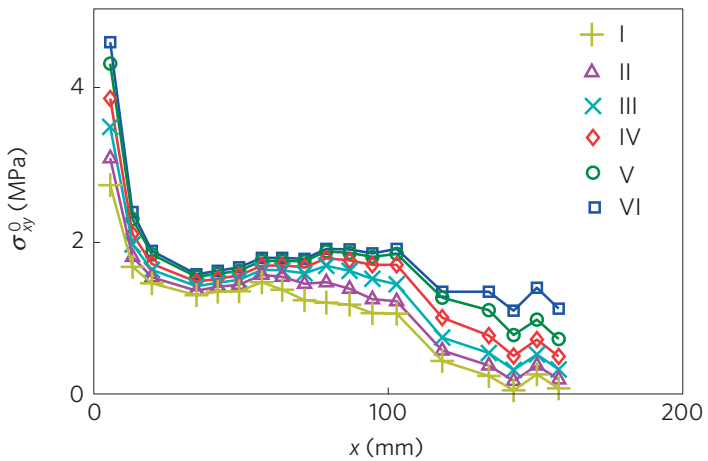

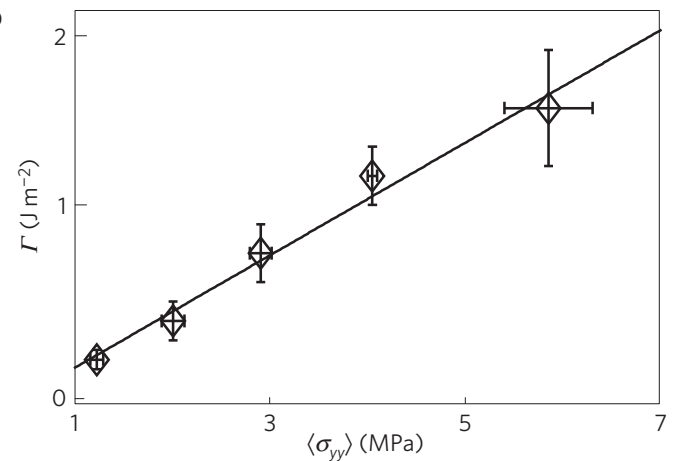

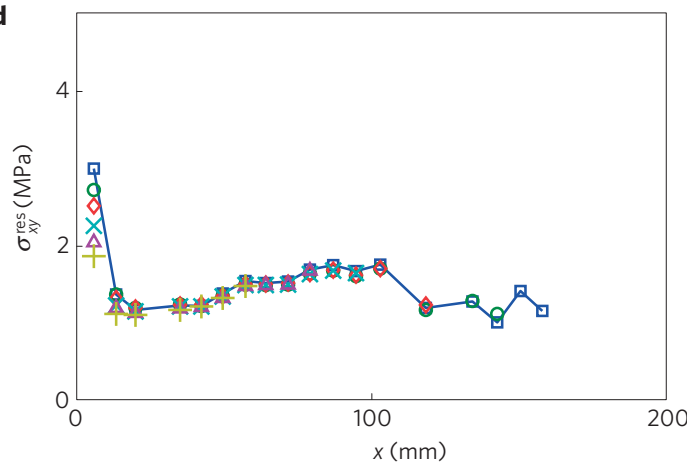

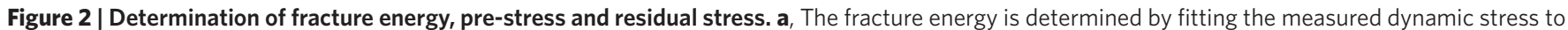
the LEFM predictions of equation (1). A typical shear stress variation $\sigma_{x y}(t)$ for a rupture propagating at velocity $v=0.04 c_{R}$ for a normal force of $F_{\mathrm{N}}=4,500 \mathrm{~N}$ at a given location along the interface $(x=65.7 \mathrm{~mm})$ is shown in red. $t=0$ corresponds to the time at which the rupture front passes the measurement point. The corresponding LEFM prediction for $\Delta \sigma_{x y}(t)$ is plotted in black. Blue lines denote the pre-stress, $\sigma_{x y}^{0}$ and the residual stress $\sigma_{x y}^{\text {res }}$ (see text). The fracture energy $\Gamma=1 \mathrm{~J} \mathrm{~m}^{-2}$ is chosen by the best fit of the three components of the stress field ${ }^{7}$. $\mathbf{b}$, Fracture energy $\Gamma$ as a function of the mean normal stress $\left\langle\sigma_{y y}\right\rangle$ as determined by fitting the stress field for different experiments as in $\mathbf{a}$. Error bars reflect the uncertainties in the determination

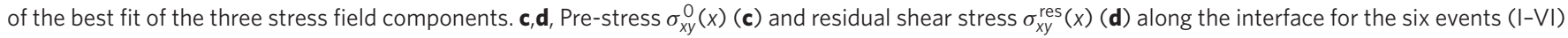
presented in Fig. 1. Note that although the residual stress beyond the arrest location of each precursor is not defined, all measured values are in excellent agreement with the values of the system-wide event VI.

equation (2), on rupture arrest, $G \rightarrow G_{\text {stat }}(l)=\alpha\left(K_{\mathrm{II}}^{2}(l, v=0) / E\right)$ and arrest will occur ${ }^{24}$ when $G_{\text {stat }}(l) \leq \Gamma$. In general, $K_{\mathrm{II}}(l, v=0) \equiv K_{\mathrm{II}}^{\text {stat }}(l)$, can be explicitly calculated ${ }^{24}$ providing $G_{\text {stat }}(l)$. For our sample geometry ${ }^{15,26}$ (Methods):

$$
K_{\mathrm{II}}^{\text {stat }}(l)=\frac{2}{\sqrt{\pi l}} \int_{0}^{l} \frac{\Delta \tau(s) F(s / l)}{\sqrt{1-(s / l)^{2}}} \mathrm{~d} s
$$

where $F(s / l)=1+0.3\left(1-(s / l)^{5 / 4}\right)$ and the stress drop for each event is defined as $\Delta \tau(x)=\sigma_{x y}^{0}(x)-\sigma_{x y}^{\text {res }}(x)$; the stress drop from the initial shear stress $\sigma_{x y}^{0}(x)$ to the residual stress $\sigma_{x y}^{\text {res }}$ at each point (Fig. 2a).

To predict the arrest location using equation (3), we need to measure both $\sigma_{x y}^{0}(x)$ and $\sigma_{x y}^{\text {res }}(x)$. Figure $2 \mathrm{c}, \mathrm{d}$ demonstrates that both quantities vary significantly in space. By definition, $\sigma_{x y}^{\text {res }}(x)$ is not defined beyond the rupture arrest location. Except very near $x=0$, where applied torques produce measurable effects ${ }^{27}$, we find that, within a given experiment, $\sigma_{x y}^{\text {res }}(x)$ is invariant from one event to another (Fig. 2d). After accounting for the edge effects (Methods), we therefore use $\sigma_{x y}^{\text {res }}(x)$ from the first system-wide event, as characteristic of the interface. We can now measure $\Delta \tau(x)$ for each event at each point $x$ (Fig. 3a). As stresses are measured slightly above the interface, we improve the accuracy of $\Delta \tau(x)$ at the interface by accounting for stress gradients (Methods).

In Fig. 3a we also present the computed value of $K_{\mathrm{II}}^{\text {stat }}(l)$ (see equation (3)) in successive events for the experiment described in Fig. 1. Predicted arrest locations, $\ell_{\text {predicted }}$, for each event are the locations where $G_{\text {stat }}=\Gamma$, where $\Gamma$ is determined by $\left\langle\sigma_{y y}\right\rangle$, the averaged value of $\sigma_{y y}$ in the section of the interface where ruptures arrest. In
Fig. $3 \mathrm{~b}$ we compare $\ell_{\text {predicted }}$ with the measured arrest length, $\ell_{\text {measured }}$, obtained from the contact area measurements. $\ell_{\text {predicted }}$ agrees well with $\ell_{\text {measured }}$. The same procedure is applied for 11 other experiments, each includes from 4 to 9 precursory events. We performed each experiment under different loading conditions for a wide range of $\Gamma$ (Fig. 4). It is clear that all of the predicted arrest lengths are in excellent agreement with the measured lengths. The variety of stress distributions and values of $\Gamma$ that are used emphasize the generality of this result. Note that this framework naturally incorporates the effects of local stress variations. The dependence of the arrest location with a heterogeneous distribution of stresses is not trivial; a given mean value of $\Delta \tau(x)$ can yield large variations in the predicted rupture arrest location, as the expression that determines $G_{\text {stat }}(l)$ generally includes a singular weight function ${ }^{24}$ (see, for example, equation (3)). For example, stress fluctuations more strongly affect the arrest if located near the arrest point.

Our results provide clear evidence that frictional rupture is really a fracture process that can be quantitatively described by fracture mechanics. The concepts presented here suggest a completely different paradigm for understanding friction from that of the classical picture, which is based on the balance of local forces (stresses). We have shown that rupture arrest is governed by energy balance precisely as described by the Griffith criterion; balancing the global (system-wide) release of elastic energy, which is embodied in the integral nature of equation (3), and the local dissipative properties of the interface governed by $\Gamma$.

Although in our experiments we considered relatively constant values of $\Gamma$, energy balance is also valid when $\Gamma$ varies along the interface. Locally high values of $\Gamma$, can, for instance, act as 


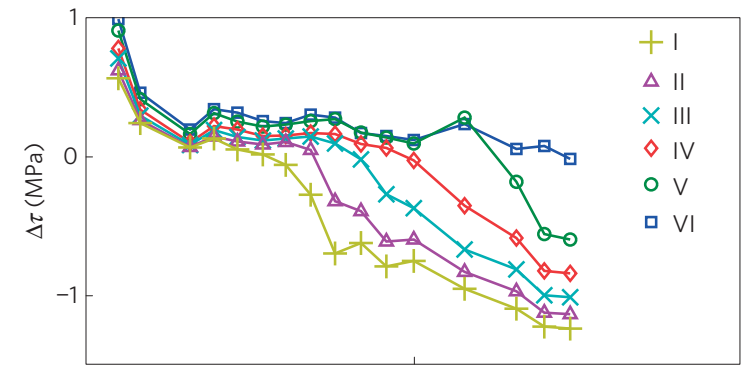

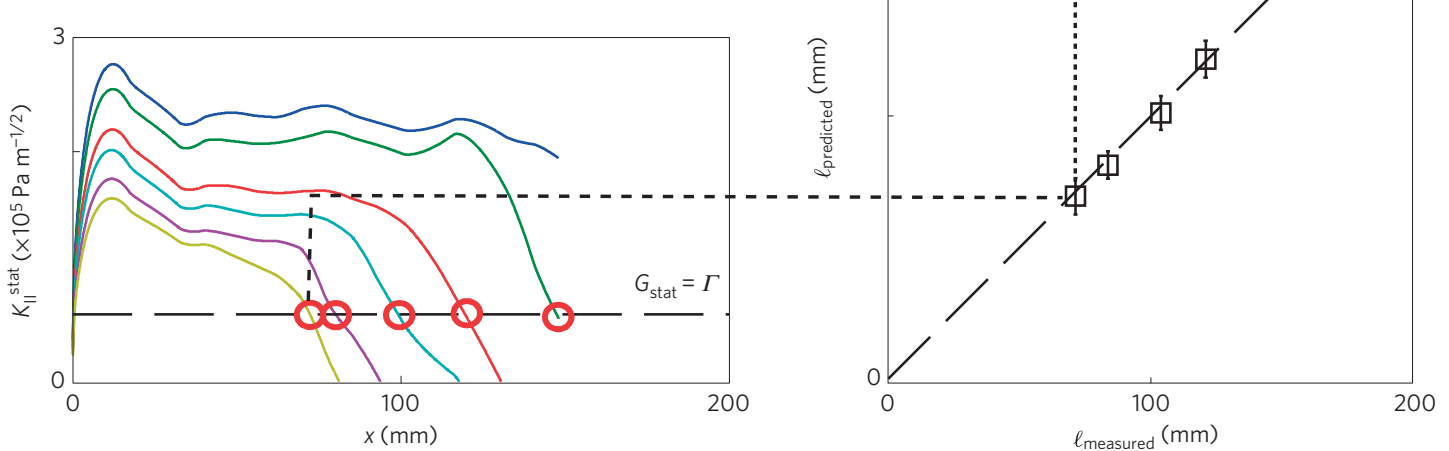

Figure 3 | Prediction of rupture arrest locations. a, (Upper panel), Shear stress drop, $\Delta \tau(x)=\sigma_{x y}^{0}(x)-\sigma_{x y}^{\text {res }}(x)$, along the interface for each of the rupture events presented in Fig. 1. Stresses are plotted for $y=0 \mathrm{~mm}$ (Methods). Data are linearly interpolated before integration. (Lower panel), The computed static stress intensity factor $K_{\|}^{\text {stat }}$ (equation (3)) along $x$ for each event from I to VI. Red circles denote the predicted location of the arrest, $x=\ell_{\text {predicted, }}$ as determined by $G_{\text {stat }}(I)=\Gamma$ (dashed line). Note that event $\mathrm{VI}$ is not arrested, being the first system-wide sliding event. $\mathbf{b}$, Comparison of the measured arrested rupture lengths, $\ell_{\text {measured, }}$ as determined from the contact area measurements to the predicted lengths, $\ell_{\text {predicted }}$ computed in $\mathbf{a}$. Examples of corresponding values of $\ell_{\text {measured }}$ and $\ell_{\text {predicted }}$ are shown by dotted lines and short-dashed line, respectively. The dashed line has a slope of 1 .

a barrier to propagation. What can cause spatial variations of $\Gamma$ ? We have shown that $\Gamma \propto \sigma_{y y}$ as the normal stress governs the geometrical size of the real contact area. Furthermore, $\Gamma$ could be affected by varying interface properties-for example, interfaces

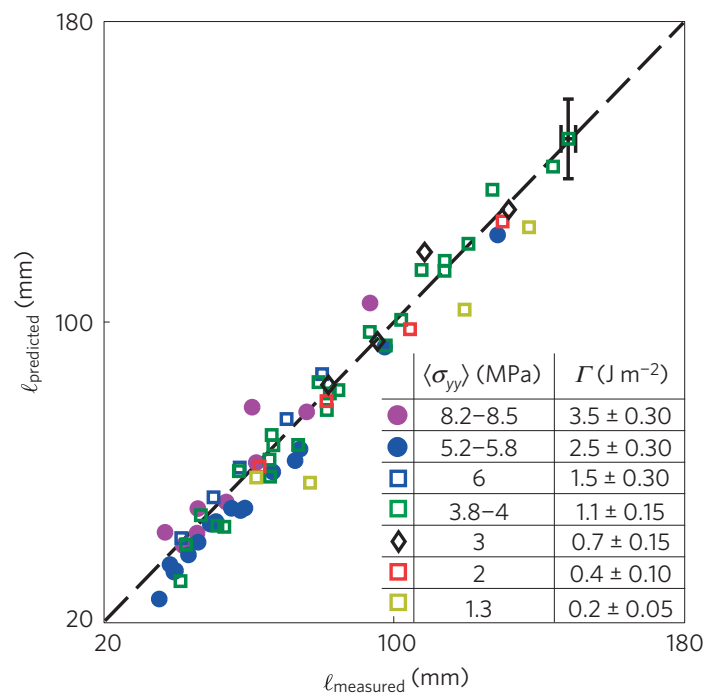

Figure 4 | Comparison of the predicted and measured rupture lengths. Predicted precursor lengths, $\ell_{\text {predicted, are compared to measured rupture }}$ lengths, $\ell_{\text {measured, }}$ for 12 experiments - each with different normal loads and stress distributions. Three such examples are presented in Fig. 1b. Symbols, as defined in Fig. 1b, indicate the following loading configurations: asymmetric geometry (filled circles), symmetric geometry without (open squares) and with stopper (open diamonds). The dashed line of slope 1 is given for reference. A typical error bar is shown. As shown in Fig. $2 b$, the fracture energy $\Gamma$ of each experiment is governed by the normal stress. incorporating different materials, chemical treatments, or pressureinduced phase transitions.

We have shown that applying fracture mechanics to friction has fundamental predictive power; knowledge of the prospective stress release at each point along the interface will tell us where a frictional rupture will arrest and, moreover, when ruptures will traverse the entire interface and precipitate frictional sliding. The initial stress profile along the interface, coupled with knowledge of the residual stress, entirely determines the eventual rupture length. Two caveats currently impede making specific predictions: knowledge of the residual stresses along the interface before an upcoming event and knowledge of when rupture nucleation will occur. The first of these might well be addressed by knowledge of the normal stress profile along the interface. Residual stresses are the manifestation of the non-broken contacts that sustain the normal load at the frictional interface. In preliminary work, we observe a strong correlation between residual shear stress and local normal stress, suggesting a local dynamic friction coefficient, but additional study is required to cement this relationship. Rupture nucleation, or the onset of friction, is a more delicate point. Previous work has shown ${ }^{5,27}$ that characteristic static friction coefficients that govern the onset of frictional motion do not exist. The Griffith criterion could, as in rupture arrest, predict rupture nucleation. The Griffith criterion, however, can be applied only when a well-defined crack tip exists. In the case of rupture arrest, a singular tip obviously exists before arrest ${ }^{7}$. Before nucleation, however, no initially sharp crack exists; a sharp initial crack must either be created or be dynamically formed. This enigmatic nucleation stage is still the subject of much active research ${ }^{20,23,28-30}$.

Although the results described above are generally relevant to every frictional interface, they are especially important to the particular question of earthquake arrest. Understanding what determines an earthquake's spatial extent is a central unresolved issue $^{18,19,21}$; either the rupture size is selected during the nucleation process or an earthquake arrests only when encountering a 
sufficiently high barrier. We have shown that the selection of the rupture length is deterministic; balancing the global stress release with local dissipation. Our results enable us to understand both viewpoints. When $\Gamma$ does not vary significantly, the initial stress profile will wholly govern rupture arrest. For example, a large drop of stress near the nucleation zone can control the arrest location. On the other hand, the existence of a sufficiently high local value of $\Gamma$ could precipitate rupture arrest by overcoming the dominance of the stress profile.

To conclude, it is far from trivial that fracture mechanics necessarily govern rupture arrest in experiments. A text book shear crack is in many ways different from frictional ruptures in a real experimental system which include significant normal stress variations, non-constant initial and residual shear stresses and effects of frictional dissipation along the crack faces. Despite these numerous and substantial complexities, fracture mechanics still provide a fundamental description of friction which is surprisingly accurate.

\section{Methods}

Methods and any associated references are available in the online version of the paper.

Received 2 July 2015; accepted 1 October 2015; published online 9 November 2015

\section{References}

1. Rubinstein, S. M., Cohen, G. \& Fineberg, J. Detachment fronts and the onset of dynamic friction. Nature 430, 1005-1009 (2004).

2. Xia, K., Rosakis, A. J. \& Kanamori, H. Laboratory earthquakes: The sub-Raleigh-to-supershear rupture transition. Science 303, 1859-1861 (2004).

3. Baumberger, T., Caroli, C. \& Ronsin, O. Self-healing slip pulses and the friction of gelatin gels. Eur. Phys. J. E 11, 85-93 (2003).

4. Ben-David, O., Cohen, G. \& Fineberg, J. The dynamics of the onset of frictional slip. Science 330, 211-214 (2010).

5. Passelègue, F. X., Schubnel, A., Nielsen, S., Bhat, H. S. \& Madariaga, R. From sub-Rayleigh to supershear ruptures during stick-slip experiments on crustal rocks. Science 340, 1208-1211 (2013).

6. Bowden, F. P. \& Tabor, D. The Friction and Lubrication of Solids 2nd edn (Oxford Univ. Press, 2001).

7. Svetlizky, I. \& Fineberg, J. Classical shear cracks drive the onset of dry frictional motion. Nature 509, 205-208 (2014).

8. Rubinstein, S. M., Cohen, G. \& Fineberg, J. Dynamics of precursors to frictional sliding. Phys. Rev. Lett. 98, 226103 (2007).

9. Maegawa, S., Suzuki, A. \& Nakano, K. Precursors of global slip in a longitudinal line contact under non-uniform normal loading. Tribol. Lett. 38, 313-323 (2010).

10. Katano, Y., Nakano, K., Otsuki, M. \& Matsukawa, H. Novel friction law for the static friction force based on local precursor slipping. Sci. Rep. 4, 6324 (2014).

11. Braun, O. M., Barel, I. \& Urbakh, M. Dynamics of transition from static to kinetic friction. Phys. Rev. Lett. 103, 194301 (2009).

12. Scheibert, J. \& Dysthe, D. K. Role of friction-induced torque in stick-slip motion. Europhys. Lett. 92, 54001 (2010).
13. Tromborg, J., Scheibert, J., Amundsen, D. S., Thogersen, K. \& Malthe-Sorenssen, A. Transition from static to kinetic friction: Insights from a 2D model. Phys. Rev. Lett. 107, 074301 (2011).

14. Radiguet, M., Kammer, D. S., Gillet, P. \& Molinari, J. F. Survival of heterogeneous stress distributions created by precursory slip at frictional interfaces. Phys. Rev. Lett. 111, 164302 (2013).

15. Kammer, D. S., Radiguet, M., Ampuero, J. P. \& Molinari, J. F. Linear elastic fracture mechanics predicts the propagation distance of frictional slip. Tribol. Lett. 57, 23 (2015).

16. Taloni, A., Benassi, A., Sandfeld, S. \& Zapperi, S. Scalar model for frictional precursors dynamics. Sci. Rep. 5, 8086 (2015).

17. Bar-Sinai, Y., Spatschek, R., Brener, E. A. \& Bouchbinder, E. Velocity-strengthening friction significantly affects interfacial dynamics, strength and dissipation. Sci. Rep. 5, 7841 (2015).

18. Ampuero, J. P., Ripperger, J. \& Mai, P. M. in Earthquakes: Radiated Energy and the Physics of Faulting Vol. 170 (eds Abercrombie, R., McGarr, A. \& Kanamori, H.) 255-261 (Geophysical Monograph Series, American Geophysical Union, 2006).

19. Ellsworth, W. L. \& Beroza, G. C. Seismic evidence for an earthquake nucleation phase. Science 268, 851-855 (1995).

20. Lapusta, N. \& Rice, J. R. Nucleation and early seismic propagation of small and large events in a crustal earthquake model. J. Geophys. Res. 108, 2205 (2003)

21. Olson, E. L. \& Allen, R. M. The deterministic nature of earthquake rupture. Nature 438, 212-215 (2005).

22. Scholz, C. H. The Mechanics of Earthquakes and Faulting 2nd edn (Cambridge Univ. Press, 2002).

23. Ben-Zion, Y. Dynamic ruptures in recent models of earthquake faults. J. Mech Phys. Solids 49, 2209-2244 (2001).

24. Freund, L. B. Dynamic Fracture Mechanics (Cambridge, 1990).

25. Palmer, A. C. \& Rice, J. R. The growth of slip surfaces in the progressive failure of over-consolidated clay. Proc. R. Soc. Lond. A 332, 527-548 (1973).

26. Tada, H., Paris, P. C. \& Irwin, G. R. The Stress Analysis of Cracks Handbook (American Society of Mechanical Engineers, 2000).

27. Ben-David, O. \& Fineberg, J. Static friction coefficient is not a material constant. Phys. Rev. Lett. 106, 254301 (2011).

28. Yang, Z. P., Zhang, H. P. \& Marder, M. Dynamics of static friction between steel and silicon. Proc. Natl Acad. Sci. USA 105, 13264-13268 (2008).

29. Kaneko, Y. \& Ampuero, J. P. A mechanism for preseismic steady rupture fronts observed in laboratory experiments. Geophys. Res. Lett. 38, L21307 (2011).

30. Latour, S., Schubnel, A., Nielsen, S., Madariaga, R. \& Vinciguerra, S. Characterization of nucleation during laboratory earthquakes. Geophys. Res. Lett. 40, 5064-5069 (2013).

\section{Acknowledgements}

We acknowledge support from the James S. McDonnell Fund, the European Research Council (Grant No. 267256) and the Israel Science Foundation (Grant 76/11). E.B.

acknowledges support from the Lady Davis Trust. We thank G. Cohen for comments.

\section{Author contributions}

E.B. and I.S. performed the measurements. All authors contributed to the analysis and writing the manuscript.

\section{Additional information}

Reprints and permissions information is available online at www.nature.com/reprints. Correspondence and requests for materials should be addressed to J.F.

\section{Competing financial interests}

The authors declare no competing financial interests. 


\section{Methods}

Experimental set-up. We used blocks of poly(methyl-methacrylate) (PMMA) with a Young's modulus $E_{\mathrm{s}}=3 \mathrm{GPa}$ for low strain rates and $E_{\mathrm{d}}=5.6 \mathrm{GPa}$ for high strain rates (see the next section) and a Poisson ratio $v=1 / 3$. Measured material wave speeds are: shear waves $c_{S}=1,345 \pm 10 \mathrm{~m} \mathrm{~s}^{-1}$, longitudinal waves

$c_{\mathrm{L}}=2,700 \pm 10 \mathrm{~m} \mathrm{~s}^{-1}$. These provide Rayleigh wave speeds $c_{\mathrm{R}}=1,237 \pm 10 \mathrm{~m} \mathrm{~s}^{-1}$ for plane stress conditions and $c_{\mathrm{R}}=1,255 \pm 10 \mathrm{~m} \mathrm{~s}^{-1}$ for plane strain conditions.

Symmetric set-up. Blocks dimensions are $200 \mathrm{~mm} \times 100 \mathrm{~mm} \times 5.5 \mathrm{~mm}(x, y, z$ directions). The contacting surfaces are optically flat. The upper surface of the top block was fixed, while the bottom block was sheared using a push-rod of dimensions $3.5 \mathrm{~mm} \times 5.5 \mathrm{~mm}$ ( $y \times z$ directions), positioned at $-3.5 \mathrm{~mm}<y<0$ and $x=0 \mathrm{~mm}$. In addition, an optional rigid stopper (of cross-section $12 \mathrm{~mm} \times 12 \mathrm{~mm}$ ) was pressed against the top block, at $x=200 \mathrm{~mm}$ and $y=11 \mathrm{~mm}$, to constrain the motion of this edge and, thereby, impose stress gradients in the $x$ and $y$ directions while a push-rod applied shear to the opposite edge. Strain field measurements were performed at 18 locations along the interface for $5.5 \mathrm{~mm}$ $<x<158.1 \mathrm{~mm}$, and $y=3.5 \mathrm{~mm}$ above the interface. Rosette-type strain gauges were spaced $7.5 \mathrm{~mm}$ apart on average $(6.5 \mathrm{~mm}<d<8.5 \mathrm{~mm})$.

Asymmetric set-up. The asymmetric set-up was constructed with a bottom block of dimensions $290 \mathrm{~mm} \times 28 \mathrm{~mm} \times 30 \mathrm{~mm}$ and a top block of dimensions $150 \mathrm{~mm} \times 100 \mathrm{~mm} \times 6 \mathrm{~mm}(x, y, z$ directions $)$. The surface of the bottom block had a $3 \mu \mathrm{m}$ r.m.s. roughness whereas the surface of the top block surface was optically flat. The upper surface of the top block was fixed and the bottom block was sheared from below by a sliding stage. A stopper, with the same characteristics as described above, prevented the displacement of the top block. Strain field measurements were performed at 15 locations along the interface for $4.3 \mathrm{~mm}$ $<x<144 \mathrm{~mm}$, and $y=3.5 \mathrm{~mm}$ above the interface. Rosette type strain gauges were spaced $10 \mathrm{~mm}$ apart on average $(7.5 \mathrm{~mm}<d<15 \mathrm{~mm})$. The resolution of the strain gauges was approximately $20-60 \mu$ strain, leading to a $0.1-0.3 \mathrm{MPa}$ resolution in the computed stress.

Transformation from strain to stress. Boundary conditions are chosen as plane stress ( $\sigma_{z z}=0$; that is, free material expansion in the $z$ direction) for the symmetric set-up and plain strain $\left(\varepsilon_{z z}=0\right.$; that is, no material expansion in the $z$ direction) for the asymmetric set-up ${ }^{7}$. The poly(methyl-methacrylate) (PMMA) is viscoelastic ${ }^{31}$. We take this effect into account while transforming strains into stresses: the static loading stresses are calculated in terms of the static Young's modulus $E_{\mathrm{s}}$ whereas the rapid drop of stress due to the rupture propagation is calculated using the dynamic Young's modulus $E_{\mathrm{d}}$. For plane stress conditions, we calculated stresses from strains as ${ }^{32}$ :

$$
\begin{gathered}
\sigma_{x y}(t)=\frac{1}{1+v}\left[E_{\mathrm{d}} \Delta \varepsilon_{x y}(t)+E_{\mathrm{s}} \varepsilon_{x y}^{0}\right] \\
\sigma_{x x}(t)=-\frac{1}{1-v^{2}}\left[E_{\mathrm{d}}\left(\Delta \varepsilon_{x x}(t)+v \Delta \varepsilon_{y y}(t)\right)+E_{\mathrm{s}}\left(\varepsilon_{x x}^{0}+v \varepsilon_{y y}^{0}\right)\right] \\
\sigma_{y y}(t)=-\frac{1}{1-v^{2}}\left[E_{\mathrm{d}}\left(\Delta \varepsilon_{y y}(t)+v \Delta \varepsilon_{x x}(t)\right)+E_{\mathrm{s}}\left(\varepsilon_{y y}^{0}+v \varepsilon_{x x}^{0}\right)\right]
\end{gathered}
$$

Calculation of the static stress intensity factor. Equation (3) is adapted from equation (8.3) from Tada ${ }^{26}$. Tada's solution provides the static stress intensity factor for a pre-existing crack of length $l$, in a plate with a free vertical boundary at $x=0$, when stresses are applied to the crack's faces. We obtain equation (3) by superposition ${ }^{24,26}$; subtracting the measured pre-stress along the prospective crack's path from Tada's solution. As a result, equation (3) calculates the stress intensity factor for a virtual crack of length $l$, as a function of the pre-existing stresses along the virtual crack's path. This formulation is conceptually equivalent to the well-known Eshelby's integral (Freund's equation (6.4.31); ref. 24) but incorporates the free boundary at $x=0$.

Definition of residual stresses. We have shown that the residual shear stress following a rupture event is roughly constant for a given experiment (Fig. 2d). Nevertheless, the top block rotates slightly during the shear loading ${ }^{27}$ and thereby induces a variation of the normal and residual stresses. This effect is largest at the interface corner $x=0$. To account for this effect, we define the residual stress of a precursor event $n$ as follows:

$$
\sigma_{x y, n}^{\mathrm{res}}(x)= \begin{cases}\sigma_{x y, n}^{\mathrm{res}}(x), & x_{1 \mathrm{st} \mathrm{SG}}<x<x_{\text {arrest }} \\ \sigma_{x y, N}^{\mathrm{res}}(x), & x_{\text {arrest }}<x<L\end{cases}
$$

where $\sigma_{x y, n}^{\text {res }}$ and $\sigma_{x y, N}^{\text {res }}$ are respectively the residual stress of the event $n$ and the main event $N$ (system-wide event); $x_{1 \mathrm{st} \mathrm{SG}}$ is the location of the first strain gauge along the interface ( $x_{1 \mathrm{st} \mathrm{SG}} \sim 5 \mathrm{~mm}$ for both set-ups). The stress is linearly extrapolated from $x=0$ to $x=x_{1 s t S G}$. Using this definition, we established the stress that would be released by a crack for any $x$ along the interface for each $n$th event, $\Delta \tau(x)=\sigma_{x y, n}^{\text {pre }}(x)-\sigma_{x y, n}^{\text {res }}(x)$ (Fig. 3a).

Taylor's expansion of stresses on the interface. As stresses are measured at $y=3.5 \mathrm{~mm}$ above the interface, and as strong gradients of stresses due to the inhomogeneous loading may take place, stresses above the interface are not a perfect measure of the stresses on the interface. To correct for the finite height of the strain gauge placement, we used a Taylor's expansion to improve our on-fault stress measurements. At equilibrium ${ }^{32}, \partial \sigma_{i k} / \partial x_{k}=0 \Rightarrow \partial \sigma_{x y} / \partial y=-\left(\partial \sigma_{x x} / \partial x\right)$. This leads to $\sigma_{x y}(0)=\sigma_{x y}\left(y_{\mathrm{SG}}\right)+y_{\mathrm{SG}}\left(\partial \sigma_{x x} / \partial x\right)$, where $y_{\mathrm{SG}}=3.5 \mathrm{~mm}$ is the position of the strain gauges and $y=0$ defines the interface. The correction is typically around $5-10 \%$ of the stress nominal value. The use of this correction does not significantly change the results, but it does reduce their scatter.

\section{References}

31. Read, B. E. \& Duncan, J. C. Measurement of dynamic properties of polymeric glasses for different modes of deformation. Polym. Test. 2, 135-150 (1981).

32. Landau, L. D., Pitaevski, L. P., Lifschitz, E. M. \& Kosevich, A. M. Theory of Elasticity 3rd edn, Vol. 7 (Pergamon, 1986). 